\title{
Comparative integromics on Eph family
}

\author{
MASUKO KATOH $^{1}$ and MASARU KATOH ${ }^{2}$ \\ ${ }^{1}$ M\&M Medical BioInformatics, Hongo 113-0033; ${ }^{2}$ Genetics and Cell Biology Section, \\ National Cancer Center Research Institute, Tokyo 104-0045, Japan \\ Received January 18, 2006; Accepted February 15, 2006
}

\begin{abstract}
EPHA1, EPHA2, EPHA3, EPHA4, EPHA5, EPHA6, EPHA7, EPHA8, EPHA10, EPHB1, EPHB2, EPHB3, EPHB4 and EPHB6 are EPH family receptors for Ephrin family ligands. Ephrin/EPH signaling pathway networks with the WNT signaling pathway during embryogenesis, tissue regeneration, and carcinogenesis. TCF/LEF-binding sites within the promoter region of human $E P H$ family members were searched for by using bioinformatics and human intelligence. Because five TCF/LEF-binding sites were identified within the 5'-promoter region of the EPHA7 gene, comparative genomics analyses on EPHA7 orthologs were further performed. EPHA7-MANEA-FHL5 locus at human chromosome 6q16.1 and EPHA10-MANEAL-FHL3 locus at human chromosome 1p34.3 were paralogous regions within the human genome. Human EPHA7 mRNA was expressed in embryonic stem (ES) cells, neural tissues, duodenal cancer and parathyroid tumors, while mouse Epha7 mRNA was expressed in fertilized egg, Rathke's pouche, visual cortex, pituitary gland, other neural tissues, pancreas, lung tumors and mammary tumors. The chimpanzee EPHA7 gene and cow Epha7 gene were identified within NW_107969.1 and AC155055.2 genome sequences, respectively. Five TCF/ LEF-binding sites within human EPHA7 promoter were conserved in the chimpanzee EPHA7 promoter, and three TCF/LEF-binding sites in the cow Epha7 promoter, but none in the mouse Epha7 promoter. Primates and cow EPHA7 orthologs were identified as evolutionarily conserved targets of the WNT/ß-catenin signaling pathway. D6S1056 microsatellite marker within EPHA7 gene is deleted in prostate cancer. Deletion and/or promoter $\mathrm{CpG}$ hypermethylation could explain the EPHA7 down-regulation in human tumors. EPHA7 is a target of systems medicine, especially in the fields of regenerative medicine and oncology.
\end{abstract}

Correspondence to: Dr Masaru Katoh, Genetics and Cell Biology Section, National Cancer Center Research Institute, 5-1-1 Tsukiji, Chuo-ku, Tokyo 104-0045, Japan

E-mail:mkatoh@ncc.go.jp

Key words: bioinformatics, comparative genomics, comparative proteomics, EFN, EPH, WNT, integrome network, systems medicine

\section{Introduction}

EPH family members are receptors for Ephrin family ligands (1-4). EPHA1, EPHA2, EPHA3, EPHA4, EPHA5, EPHA6, EPHA 8 and EPHA10 are classified as EPHA subfamily members, while EPHB1, EPHB2, EPHB3, EPHB4 and EPHB6 are classified as the EPHB subfamily members. EPH family members consist of extracellular Ephrin-binding domain, cysteine-rich domain, two fibronectin type III repeats as well as cytoplasmic tyrosine kinase domain and C-terminal SAM motif.

EFNA1, EFNA2, EFNA3, EFNA4, EFNA5, EFNB1, EFNB2 and EFNB3 are EFRIN (EFN) family ligands for EPH family receptors (1-4). EFNA1, EFNA2, EFNA3, EFNA4 and EFNA5 are EFNA subfamily members characterized as GPIanchored cell-surface proteins with EPH-binding domain, while EFNB1, EFNB2 and EFNB3 are EFNB subfamily members characterized as transmembrane proteins with an extracellular EPH-binding domain and a cytoplasmic PDZbinding motif.

Ephrin/EPH signaling pathway networks with WNT signaling pathway in a variety of processes, such as axon guidance and gastrointestinal morphogenesis, during embryogenesis, tissue regeneration and carcinogenesis (5). Canonical WNT signaling pathway activates the transcription of target genes, such as DKK1,DKK4, FGF18 and FGF20, depending on the transcriptional complex consisting of TCF/LEF, $\beta$ catenin, BCL9/BCL9L and PYGO1/PYGO2 (6-22).

TCF/LEF-binding sites within the promoter region of human $E P H$ family members were searched for by using bioinformatics and human intelligence. Because five TCF/LEFbinding sites were identified within the 5'-promoter region of the EPHA7 gene, comparative genomics analyses on EPHA7 orthologs were further performed.

\section{Materials and methods}

WNT target gene screening. Genome sequences corresponding to human EPHA1, EPHA2, EPHA3, EPHA4, EPHA5, EPHA6, EPHA8, EPHA10, EPHB1, EPHB2, ЕPHB3, ЕPHB4 and EPHB6 genes were searched for with BLAST programs (http:// www.ncbi.nlm.nih.gov) as described previously (23-28). TCF/ LEF-binding sites within the 5'-flanking promoter region of the above genes were searched for based on bioinformatics and manual inspection as described previously $(29,30)$. 
A

\begin{tabular}{|c|c|c|c|}
\hline $\begin{array}{c}\text { Human } \\
\text { gene }\end{array}$ & $\begin{array}{c}\text { Human } \\
\text { complete } \mathrm{CDS}\end{array}$ & $\begin{array}{l}\text { Human genome clone } \\
\text { including promoter }\end{array}$ & $\begin{array}{l}\text { TCF } / E F \text { binding site } \\
\text { within human promoter }\end{array}$ \\
\hline EPHA 1 & NM_005232.3 & $A C 092214.3$ & 0 \\
\hline EPHA2 & NM-004431.2 & AL 451042.10 & 1 \\
\hline EPHA3 & NM_-005233.3 & $\mathrm{AC138973.2}$ & 2 \\
\hline $\begin{array}{l}E P H A 4 \\
E P H A\end{array}$ & NM_-004438.3 & Ac079834.8 & 0 \\
\hline EPHA 5 & $N M-004439 ; 4$ & AC104137.5 & 2 \\
\hline EPHA 6 & АКำ92565.1 & AC109782.7 & 0 \\
\hline EPHA & NM_004440.2 & AL121966.10 & 5 \\
\hline $\begin{array}{l}\text { EPHAS } \\
\text { EPHD }\end{array}$ & NM $020526:^{3}$ & AL 035703.21 & 0 \\
\hline $\begin{array}{l}E P H A 10 \\
E P H B 1 \\
E P H A\end{array}$ & NM $00444 i^{1} .3$ & $\begin{array}{l}\mathrm{AC} 104336.2 \\
\mathrm{ACO} 016931.21\end{array}$ & ${ }_{0}^{2}$ \\
\hline EPHB 2 & $\mathrm{NM}-017449.2$ & AL.158086.32 & 0 \\
\hline EPHB3 & $\mathrm{NM}_{-} 004443.3$ & $\mathrm{AC} 112643.12$ & 1 \\
\hline EPHB & NM_004444.4 & $A F 312032.1$ & 0 \\
\hline EPHB 6 & $\mathrm{NM}_{-} 004445.2$ & AC104597.3 & 2 \\
\hline
\end{tabular}

B

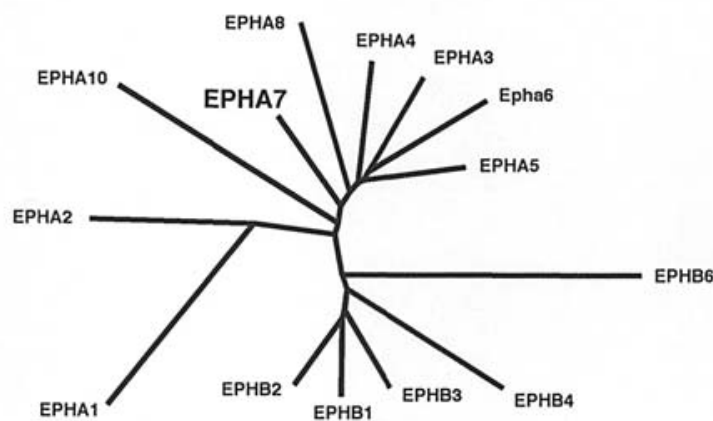

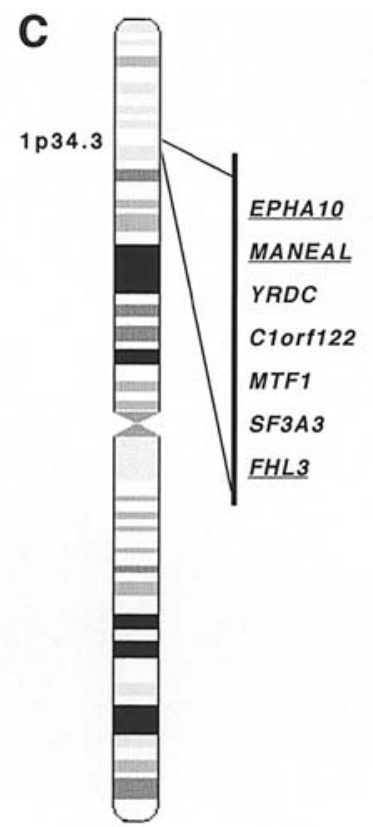

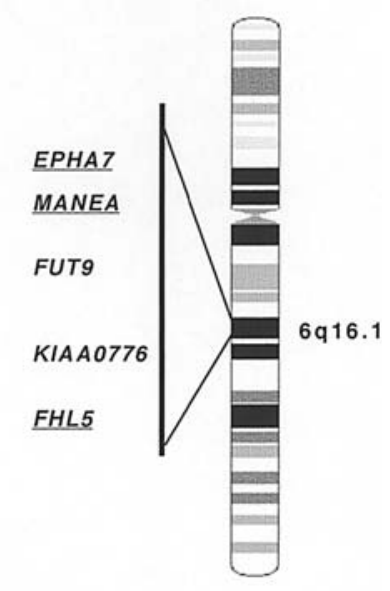

Figure 1. (A), The human EPH gene family. Gene symbol, complete coding sequence, genome sequence and the number of TCF/LEF-binding sites within the promoter region of $14 \mathrm{EPH}$ family genes are listed. Five TCF/LEF-binding sites exist within human EPHA7 promoter. (B), Phylogenetic analysis on the EPH family. EPHA7 is more related to EPHA8 and EPHA10 than the other members of the EPHA family. (C), Intra-species comparative genomics on EPHA7 and EPHA10 loci. EPHA7-MANEA-FHL5 locus at human chromosome 6q16.1 and EPHA10-MANEAL-FHL3 locus at human chromosome 1p34.3 are paralogous regions within the human genome.

Identification of the chimpanzee and cow EPHA7 orthologs. Chimpanzee and cow genome sequences homologous to human EPHA7 were searched for with BLAST programs as described previously (31-36). TCF/LEF-binding sites within the 5'-flanking promoter region of EPHA7 orthologs were also searched for.

Comparative proteomics analysis. Phylogenetic analysis on EPH family proteins was performed by using the CLUSTALW program.

Comparative genomics analyses. Phylogenetic analysis on the promoter of EPHA7 orthologs was performed by using the CLUSTALW program. Promoter region of human, chimpanzee and cow EPHA7 orthologs were aligned by using the Genetyx program and manual curation.

In silico expression analyses. Expressed sequence tags (ESTs) derived from human EPHA7 gene and mouse Epha7 gene were searched for by using the BLAST programs. The sources of human EPHA7 ESTs and those of mouse Epha7 ESTs were listed up for in silico expression analyses.

\section{Results}

Screening of TCF/LEF-binding site within promoter region of EPH family genes. Human EPHA1 RefSeq (NM_005232.3), EPHA2 RefSeq (NM_004431.2), EPHA3 RefSeq (NM_ 005233.3), EPHA4 RefSeq (NM_004438.3), EPHA5 RefSeq (NM_004439.4), EPHA6 RefSeq (AK092565.1), EPHA7 RefSeq (NM_004440.2), EPHA8 RefSeq (NM_020526.3), EPHA10 coding sequence (AJ872185.1), EPHB1 RefSeq (NM_004441.3), EPHB2 RefSeq (NM_017449.2), EPHB3
RefSeq (NM_004443.3), EPHB4 RefSeq (NM_004444.4) and EPHB6 RefSeq (NM_004445.2) were used as query sequences for the BLAST programs to identify genome clones corresponding to $E P H$ family genes. The 5'-flanking promoter region of human EPHA1, EPHA2, EPHA3, EPHA4, EPHA5, EPHA6, ЕPHA8, EPHA10, ЕPHB1, ЕPHB2, ЕPHB3, ЕPHB4 and EPHB6 genes were identified within AC092214.3, AL451042.10, AC138973.2, AC079834.8, AC104137.5, AC109782.7, AL121966.10, AL035703.21, AC104336.2, AC016931.21, AL158086.32, AC112643.12, AF312032.1 and AC104597.3 genome sequences, respectively (Fig. 1A). TCF/ LEF-binding sites within the 5'-promoter region of human $E P H$ family genes were then searched for based on manual inspection. Five TCF/LEF-binding sites were identified within the human EPHA7 promoter (Fig. 1A).

Comparative integromics analysis on EPHA7. Comparative proteomics analysis on EPHA7 was at first performed. Because human EPHA6 RefSeq encoded a C-terminally truncated isoform, mouse Epha6 was used for the phylogenetic analysis in this study. Phylogenetic analysis revealed that EPHA7 was more related to EPHA8 and EPHA10 than the other EPHA subfamily members (Fig. 1B).

Intra-species comparative genomics analysis was next performed. MDN1, CASP8AP2, CX62, BACH2 and MAP3K7 genes were located centromeric to EPHA7 gene, while MANEA, FUT9, KIAA0776 and FHL5 were located telomeric to EPHA7 gene. Paralogs corresponding to these genes around the EPHA7 locus were searched for by using the BLAST programs. MANEA-like (MANEAL) and FHL5 genes, located around the EPHA10 locus at human chromosome 1p34.3, were paralogs of MANEA and FHL3 genes, respectively. These facts indicate that EPHA7-MANEA-FHL5 locus at human chromosome 
A

\begin{tabular}{lllc}
\hline $\begin{array}{c}\text { EPHA7 } \\
\text { ortholog }\end{array}$ & RefSeq & $\begin{array}{c}\text { Genome clone } \\
\text { including promoter }\end{array}$ & $\begin{array}{c}\text { TCF/EF binding site } \\
\text { within promoter }\end{array}$ \\
\hline Hs EPHA7 & NM_004440.2 & AL121966.10 & 5 \\
Pt EPHA7 & Not determined & NW_107969.1 & 5 \\
Bt EPha7 & Not determined & AC155055.2 & 3 \\
Mm Epha7 & NM_010141.2 & BX000989.10 & 0 \\
Rn Epha7 & NM_134331.1 & AC106555.4 & 1 \\
\hline
\end{tabular}

B

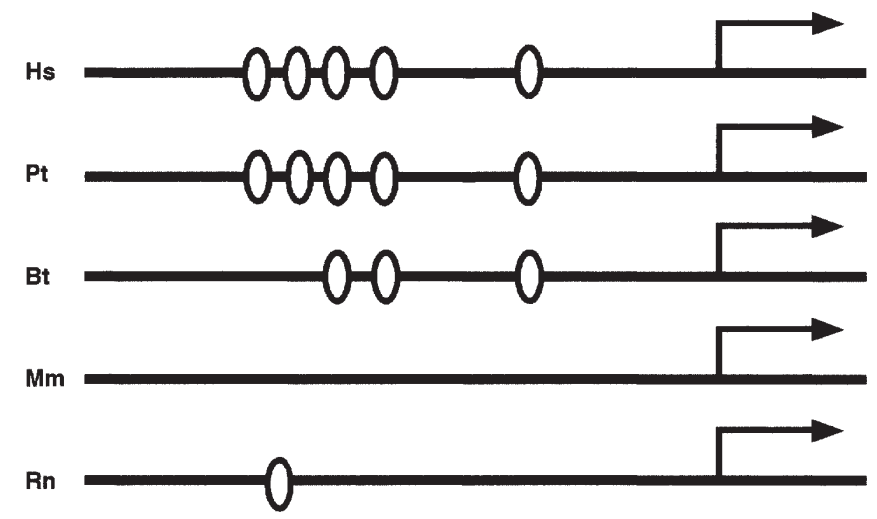

C

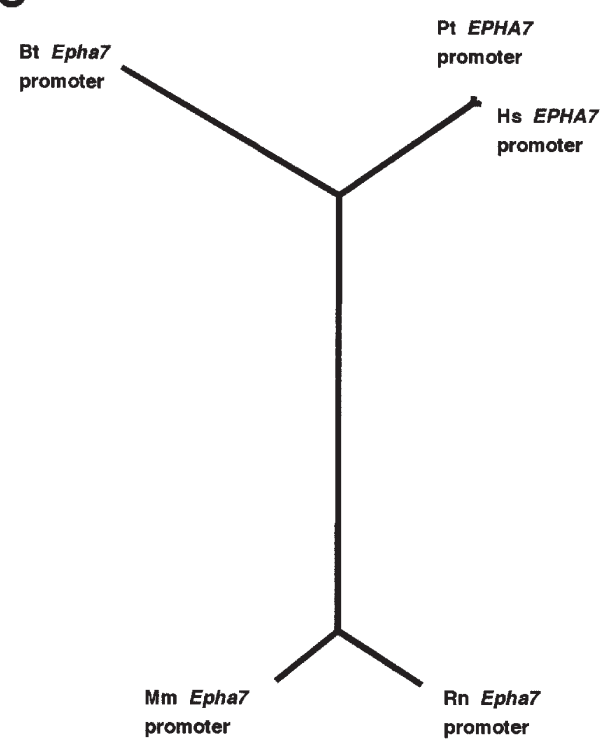

Figure 2. Inter-species comparative genomics analyses on EPHA7 promoters. Hs, human; Pt, chimpanzee; Bt, cow; Mm, mouse; Rn, rat. (A), Promoter of mammalian EPHA7 orthologs. (B), Schematic representation of mammalian EPHA7 promoters. TCF/LEF-binding sites are shown by oval symbols. (C), Phylogenetic analysis on mammalian EPHA7 promoters. Human, chimpanzee and cow EPHA7 promoters are significantly divergent from the mouse and rat Epha7 promoters.

6q16.1 and EPHA10-MANEAL-FHL3 locus at human chromosome $1 \mathrm{p} 34.3$ were paralogous regions within the human genome (Fig. 1C).

Expression profile of human EPHA7 and mouse Epha7 mRNAs. In silico expression analyses were performed to compare the expression profile of human EPHA7 and mouse Epha7 mRNAs. Human EPHA7 mRNA was expressed in embryonic stem (ES) cells, neural tissues, duodenal cancer and parathyroid tumors, while mouse Epha7 mRNA was expressed in fertilized egg, Rathke's pouche, visual cortex, pituitary gland, other neural tissues, pancreas, lung tumors and mammary tumors.

Identification of the chimpamzee and cow EPHA7 orthologs. BLAST programs using human EPHA7 RefSeq revealed that chimpanzee EPHA7 gene was located within NW_107969.1 genome sequence. Exon-intron boundaries of chimpanzee EPHA7 gene were determined based on the consensus sequence of exon-intron junctions. Although 3'-part of exon 8 was located within the sequence gap, chimpanzee EPHA7 gene was found consisting of 17 exons.

BLAST programs using human EPHA7 RefSeq revealed that exons 1-3 of cow Epha7 gene were located within the AC155055.2 genome sequence.

Comparative genomics analyses on EPHA7 promoters. Human EPHA7 promoter, chimpanzee EPHA7 promoter and cow Epha7 promoter were located within AL121966.10, NW_107969.1 and AC155055.2 genome sequences, respectively, as mentioned above. BLAST programs revealed that mouse and rat Epha7 promoters were located within BX000989.10 and AC106555.4 genome sequences, respectively (Fig. 2A). GC content of human, chimpanzee and cow EPHA7 promoters were $46.3 \%$, that of mouse Epha7 promoter was $50.4 \%$, and that of rat Epha7 promoter was $48.0 \%$.

Five TCF/LEF-binding sites within human EPHA7 promoter were located about $1200 \mathrm{bp}, 1150 \mathrm{bp}, 1000 \mathrm{bp}$, $900 \mathrm{bp}$, and $550 \mathrm{bp}$ upstream of the transcription start site (Fig. 2B). Five TCF/LEF-binding sites within human EPHA7 promoter were completely conserved in the chimpanzee EPHA7 promoter. Although the first two TCF/LEF-binding sites within human EPHA7 promoter have undergone nucleotide substitutions, the third to fifth TCF/LEF-binding sites within human EPHA7 promoter were completely conserved in the cow Epha7 promoter. On the other hand, the $\mathrm{TCF} / \mathrm{LEF}-$ binding site was not identified within the mouse Epha7 promoter (Fig. 2B).

Phylogenetic analysis revealed that human, chimpanzee and cow EPHA7 promoters were completely divergent from mouse and rat Epha7 promoters (Fig. 2C). Alignment of human, chimpanzee and cow EPHA7 promoters revealed that the 5'-promoter region of human, chimpanzee and cow EPHA7 orthologs with multiple TCF/LEF sites were well conserved (Fig. 3). Based on these facts, it was concluded that primates and cow EPHA7 orthologs were evolutionarily conserved targets of the WNT/ß-catenin signaling pathway.

\section{Discussion}

TCF/LEF-binding sites within the promoter region of human EPHA1, ЕPHA2, ЕPHA3, ЕPHA4, ЕPHA5, ЕPHA6, ЕPHA8, EPHA10, EPHB1, EPHB2, EPHB3, EPHB4 and EPHB6 genes were searched for in this study. Because five TCF/LEFbinding sites were identified within the 5'-promoter region of EPHA7 gene at human chromosome 6q16.1 (Fig. 1A), comparative genomics analyses on EPHA7 orthologs were 


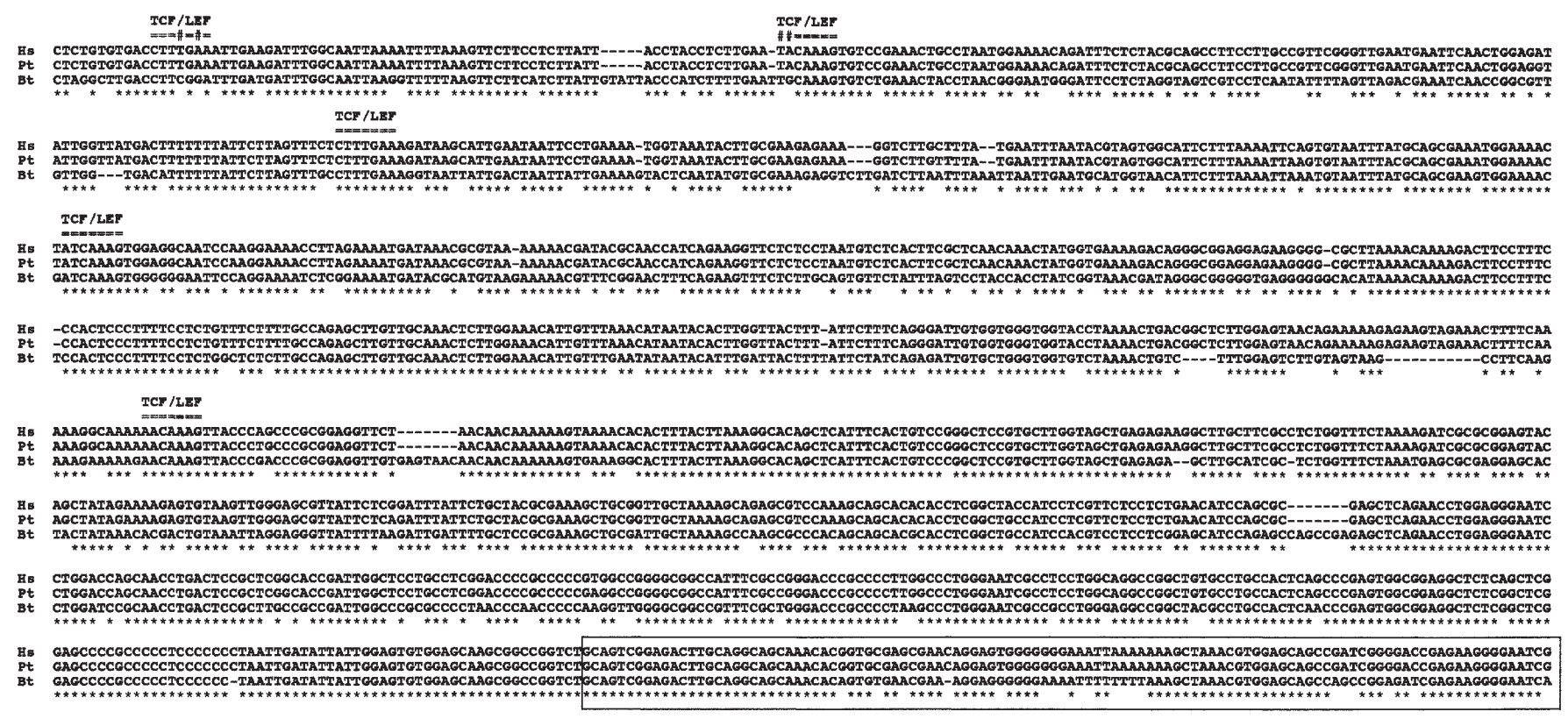

Figure 3. Alignment of human, chimpanzee and cow EPHA7 promoters. Hs, human; Pt, chimpanzee; Bt, cow. Region corresponding to exon 1 of human EPHA7 gene is boxed. Five TCF/LEF-binding sites, conserved in primate EPHA7 promoters, are shown by double over-lines. Nucleotide changes to disrupt the conserved TCF/LEF-binding sites in cow Epha7 promoter are shown by a sharp. Primates and cow EPHA7 promoters with multiple TCF/LEF-binding sites are well conserved.

further performed. EPHA7-MANEA-FHL5 locus at human chromosome 6q16.1 and EPHA10-MANEAL-FHL3 locus at human chromosome 1p34.3 were paralogous regions within the human genome (Fig. 1C).

The chimpanzee EPHA7 and cow Epha7 genes were identified within NW_107969.1 and AC155055.2 genome sequences, respectively (Fig. 2A). Five TCF/LEF-binding sites within human EPHA7 promoter were conserved in chimpanzee EPHA7 promoter, and three TCF/LEF-binding sites in the cow Epha7 promoter, but none in the mouse Epha7 promoter (Fig. 2B). Human EPHA7 mRNA and rodent Epha7 mRNA were expressed in early embryonic cells, neural tissues and several tumor types; however, primate EPHA7 promoters were significantly divergent from the rodent Epha7 promoters (Fig. 2C). More detailed comparison on the expression profile of human EPHA7 mRNA and rodent Epha7 mRNA should be performed in the future.

Primates and cow EPHA7 orthologs were identified as evolutionarily conserved targets of the $\mathrm{WNT} / \mathrm{B}$-catenin signaling pathway. Although WNT/ß-catenin signaling pathway is activated in most cases of human colorectal cancer (37), expression of EPHA7 mRNA in human colorectal cancer was not detected by in silico expression analyses. Ephrin/EPH signaling, implicated in the maintenance of colorectal mucosal homeostasis, is down-regulated in mouse advanced colorectal cancer $(5,38)$. Microsatellite markers D6S1942, D6S1293 and D6S1056 were identified within the human EPHA7 gene in this study. Among these microsatellite markers, D6S1056 is deleted in prostate cancer (39). EPHA7 might be down-regulated in colorectal cancer due to a deletion. Alternatively, EPHA7 mRNA might be down-regulated in extra-neural tissues due to promoter $\mathrm{CpG}$ hypermethylation.
Because EPHA7 is an evolutionarily conserved target of the $\mathrm{WNT} / \mathrm{\beta}$-catenin signaling pathway at least in primates and cow, EPHA7 is a target of systems medicine, especially in the fields of regenerative medicine and oncology.

\section{References}

1. Flanagan JG and Vanderhaeghen P: The ephrins and Eph receptors in neural development. Annu Rev Neurosci 21: 309-345, 1998.

2. Holder $\mathrm{N}$ and Klein R: Eph receptors and ephrins: effectors of morphogenesis. Development 126: 2033-2044, 1999.

3. Wilkinson DG: Multiple roles of Eph receptors and ephrinin neural development. Nat Rev Neurosci 2: 155-164, 2001

4. Mancia F and Shapiro L: ADAM and Eph: how Ephrin-signaling cells become detached. Cell 123: 185-187, 2005.

5. Battle E, Henderson JT, Beghtel H, et al: B-catenin and TCF mediate cell positioning in the intestinal epithelium by controlling the expression of EphB/EphrinB. Cell 111: 251-263, 2002.

6. Katoh M: WNT and FGF gene clusters. Int J Oncol 21: 1269-1273, 2002.

7. Katoh M: Regulation of WNT signaling molecules by retinoic acid during neuronal differentiation in NT2 cells: threshold model of WNT action. Int J Mol Med 10: 683-687, 2002.

8. Heller RS, Klein T, Ling Z, Heimberg H, Katoh M, Madsen OD and Serup P: Expression of WNT, Frizzled, $s F R P$, and $D K K$ genes in adult human pancreas. Gene Expr 11: 141-147, 2003.

9. Katoh M: WNT2 and human gastrointestinal cancer. Int J Mol Med 12: 811-816, 2003

10. Katoh $\mathrm{M}$ and Katoh M: Identification and characterization of human $B C L 9 L$ gene and mouse $B c l 9 l$ gene in silico. Int J Mol Med 12: 643-649, 2003

11. Garciadiego-Cazares D, Rosales C, Katoh M and ChimalMonroy J: Coordination of chondrocyte differentiation and joint formation by $\alpha 5 \beta 1$ integrin in the developing appendicular skeleton. Development 131: 4735-4742, 2004.

12. Katoh $\mathrm{M}$ and Katoh M: Comparative genomics on WNT8A and WNT8B genes. Int J Oncol 26: 1129-1133, 2005.

13. Katoh M: Molecular evolution of $W N T 2 B$ orthologs. Int J Oncol 26: 1135-1139, 2005

14. Katoh M: Comparative genomics on WNT3-WNT9B gene cluster. Int J Mol Med 15: 743-747, 2005. 
15. Swain RK, Katoh M, Medina A and Steinbeisser H: Xenopus frizzled-4S, a splicing variant of Xfz4, is a context-dependent activator and inhibitor of Wnt/ß-catenin signaling. Cell Commun Signal 3: 12, 2005.

16. Katoh M: Epithelial-mesenchymal transition in gastric cancer. Int J Oncol 27: 1677-1683, 2005.

17. Katoh M: WNT2B: comparative integromics and clinical application. Int J Mol Med 16: 1103-1108, 2005.

18. Katoh Y and Katoh M: Comparative genomics on DKK1 orthologs. Int J Oncol 27: 275-279, 2005.

19. Katoh Y and Katoh M: Comparative genomics on $D K K 2$ and DKK4 orthologs. Int J Mol Med 16: 477-481, 2005.

20. Katoh M and Katoh M: Comparative genomics on FGF20 orthologs. Oncol Rep 14: 287-290, 2005.

21. Katoh $\mathrm{M}$ and Katoh $\mathrm{M}$ : Comparative genomics on $F G F 8$, $F G F 17$, and FGF 18 orthologs. Int J Mol Med 16: 493-496, 2005.

22. Katoh Y and Katoh M: Comparative genomics on FGF16 orthologs. Int J Mol Med 16: 959-963, 2005.

23. Katoh M: Paradigm shift in gene-finding method: from benchtop approach to desk-top approach. Int J Mol Med 10: 677-682, 2002.

24. Katoh M and Katoh M: Evolutionary conservation of CCND1ORAOVI-FGF19-FGF4 locus from zebrafish to human. Int J Mol Med 12: 45-50, 2003

25. Katoh $\mathrm{M}$ and Katoh M: $C L D N 23$ gene, frequently down-regulated in intestinal-type gastric cancer, is a novel member of CLAUDIN gene family. Int J Mol Med 11: 683-689, 2003.

26. Katoh $M$ and Katoh M: Identification and characterization of human MPP7 gene and mouse Mpp7 gene in silico. Int J Mol Med 13: 333-338, 2004.

27. Katoh $M$ and Katoh M: Identification and characterization of Crumbs homolog 2 gene at human chromosome 9q33.3. Int J Oncol 24: 743-749, 2004.
28. Katoh $\mathrm{M}$ and Katoh M: Identification and characterization of human HES2, HES3, and HES5 genes in silico. Int J Oncol 25: 529-534, 2004.

29. Katoh Y and Katoh M: Comparative genomics on HHIP family orthologs. Int J Mol Med 17: 391-395, 2006.

30. Katoh Y and Katoh M: FGF signaling inhibitor, SPRY4, is evolutionarily conserved target of WNT signaling pathway in progenitor cells. Int J Mol Med 17: 529-532, 2006.

31. Katoh $\mathrm{M}$ and Katoh $\mathrm{M}$ : Identification and characterization of human HESL, rat Hesl and rainbow trout hesl genes in silico. Int J Mol Med 14: 747-751, 2004

32. Katoh $\mathrm{M}$ and Katoh M: Comparative genomics on WNT5A and WNT5B genes. Int J Mol Med 15: 749-753, 2005.

33. Katoh Y and Katoh M: Comparative genomics on WNT11 gene. Int J Mol Med 15: 879-883, 2005.

34. Katoh Y and Katoh M: Comparative genomics on VANGL1 and VANGL2 genes. Int J Oncol 26: 1435-1440, 2005.

35. Katoh Y and Katoh M: Comparative genomics on SFRP1 orthologs. Int J Oncol 27: 861-865, 2005.

36. Katoh Y and Katoh M: WNT antagonist, SFRP1, is Hedgehog signaling target. Int J Mol Med 17: 171-175, 2006.

37. Morin PJ, Sparks AB, Korinek V, et al: Activation of B-cateninTcf signaling in colon cancer by mutations in $\beta$-catenin or APC. Science 275: 1787-1890, 1997.

38. Battle E, Bacani J, Begthel H, et al: EphB receptor activity suppresses colorectal cancer progression. Nature 435: 1126-1130, 2005.

39. Srikantan V, Sesterhenn IA, Davis L, et al: Allelic loss on chromosome $6 \mathrm{q}$ in primary prostate cancer. Int J Cancer 84 : 331-335, 1999. 